\title{
DISEÑO DE UNA VIVIENDA BIOCLIMÁTICA EN LA CIUDAD DE TUMBES
}

\author{
BIOCLIMATIC HOUSING DESIGN \\ IN TUMBES CITY
}

DIEGO PÉREZ GUERRA
Universidad de Lima

El siguiente artículo tiene como objetivo detallar el proceso de diseño de un proyecto de vivienda en la ciudad de Tumbes, Perú, haciendo énfasis en el uso de métodos pasivos de control ambiental. Se detallan los puntos de análisis necesarios para la realización de una ficha bioclimática, esencial para definir las estrategias de control pasivo que se van a utilizar. Se trata también de cómo estas estrategias se plasman en criterios y elementos arquitectónicos para lograr el confort térmico de los usuarios sin perjudicar el buen diseño y funcionamiento de la vivienda como tal.

sistemas pasivos, control ambiental, confort térmico, arquitectura bioclimática, Tumbes
Recibido: 3 de junio del 2020

Aprobado: 3 de noviembre del 2020

doi: https://doi.org/10.26439/limaq2021.n008.5557

The following article aims to detail the design process of a housing project in the city of Tumbes, Perú, emphasizing the use of passive environmental control methods. The necessary analysis points are detailed for the realization of a bioclimatic sheet, essential to define the passive control strategies to be used. It is also discussed how these strategies are reflected in criteria and architectural elements to achieve the thermal comfort of the users without damaging the good design and operation of the house as such.

passive system, environmental control, thermal comfort, bioclimatic architecture, Tumbes 


\section{INTRODUCCIÓN}

El historiador Leland M. Roth (1999) afirma que, desde su planteamiento por Vitruvio en el siglo iv a. de C., la tríada utilidad, solidez y belleza "sigue siendo un compendio válido de la buena arquitectura" (p. 9). En la actualidad, con el auge de la arquitectura sostenible, la utilidad de la arquitectura también debe reflejar el manejo de los recursos en el proyecto, tanto materiales como energéticos. En respuesta a esta nueva consideración, aparece la arquitectura bioclimática, dirigida a los siguientes principios: "Al mejoramiento de la calidad de vida de los usuarios desde el punto de vista del confort higrotérmico, a la integración del objeto arquitectónico a su contexto y a incidir en la reducción de la demanda de energía convencional" (Garzón, 2007, p. 15).

La arquitectura bioclimática considera principalmente las condiciones del terreno, el recorrido del sol, las corrientes de aire y la humedad; con base en estas condiciones se decide la orientación y la forma de la edificación. Durante el diseño se deben considerar todos los elementos del edificio en conjunto: estructuras, cerramientos, instalaciones y revestimientos. En el curso de Acondicionamiento Ambiental 1, aprendimos sobre este tipo de arquitectura y los diversos criterios bioclimáticos que considera: radiación directa, radiación difusa, orientación, temperatura, humedad, humedad y precipitaciones, vientos, obstrucciones y parasoles. Con esta información realizamos una ficha bioclimática y el diseño de una vivienda que se presenta a continuación.

\section{FICHA BIOCLIMÁTICA}

La ficha bioclimática es un resumen de la información climática de una ciudad o zona específica; incluye criterios y recomendaciones para alcanzar el confort térmico en las condiciones presentes, y permite plantear una propuesta adecuada según el contexto del proyecto. Está compuesta por data climática desplegada en diferentes cuadros y formatos, y por un análisis y recomendaciones sobre esta data.

En la parte de información, la ficha contiene cinco cuadros. El primero muestra la clasificación climatológica según Senamhi, la ubicación geográfica de la zona y una tabla de normales climatológicas que engloba las variables de temperatura, precipitaciones, humedad relativa, horas de sol y vientos predominantes, y los compara con los doce meses del año (véase la figura 1). Este cuadro sirve para identificar el mes con la mayor y menor temperatura, considerando no solo los valores máximos y mínimos en grados centígrados, sino también las demás variables ya mencionadas. Por ejemplo, en nuestro caso de estudio, para la ciudad de Tumbes pudimos señalar a marzo como el mes más caluroso, no solo porque tenía la temperatura máxima media más alta y baja humedad, sino porque también contaba con la mayor cantidad de horas de sol. A primera vista, el mes más frío sería septiembre, porque tiene la temperatura mínima media más baja de todo el año; sin embargo, concluimos que agosto es el más frío porque presenta casi las mismas temperaturas, pero con mayor humedad y muchas menos horas de sol. 
Este es un ejemplo de la importancia de analizar todos los datos y no solo las temperaturas para identificar el mes con la mayor y menor temperatura.

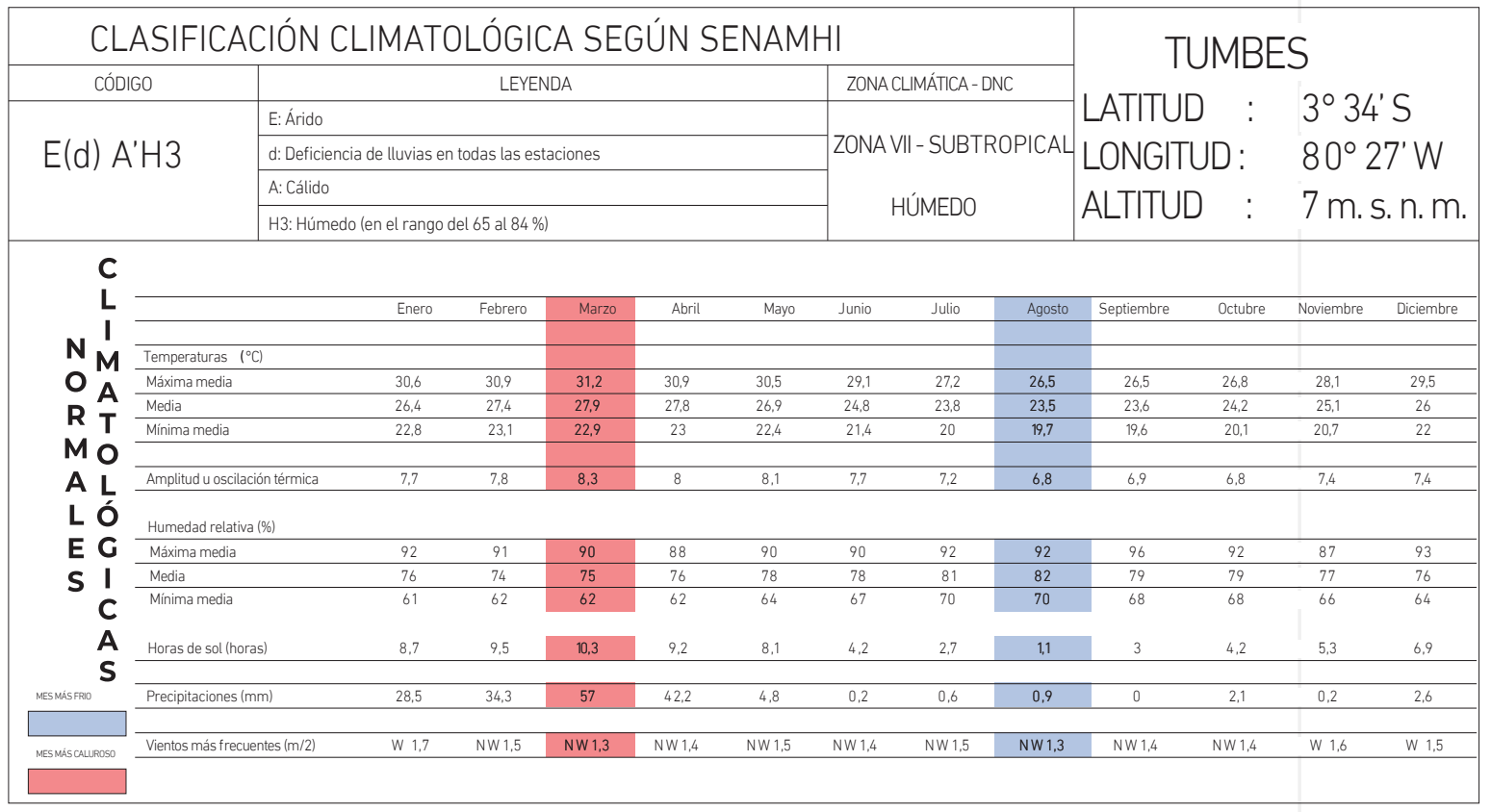

El segundo cuadro presenta el gráfico ombrotérmico, que es una comparación a lo largo del año de las temperaturas y las precipitaciones (véase la figura 2). En la parte inferior se colocan los meses, a la izquierda las temperaturas medias mensuales en grados centígrados y a la derecha las precipitaciones medias mensuales en milímetros. La escala de las precipitaciones debe ser el doble de la escala de las temperaturas, es decir, a $20{ }^{\circ} \mathrm{C}$ le corresponden $40 \mathrm{~mm}$ de precipitación, a $30^{\circ} \mathrm{C}, 60 \mathrm{~mm}$ y así sucesivamente. Esto nos permite identificar el periodo seco del año, cuando básicamente la precipitación es inferior al doble de la temperatura media. En nuestro caso, observamos que todo el año es seco, a excepción de marzo, en que las altas temperaturas identificadas en el cuadro anterior justifican el aumento de las precipitaciones.
Figura 1.

Tabla de clasificación climatológica

Elaboración propia 
Gráfico ombrotérmico

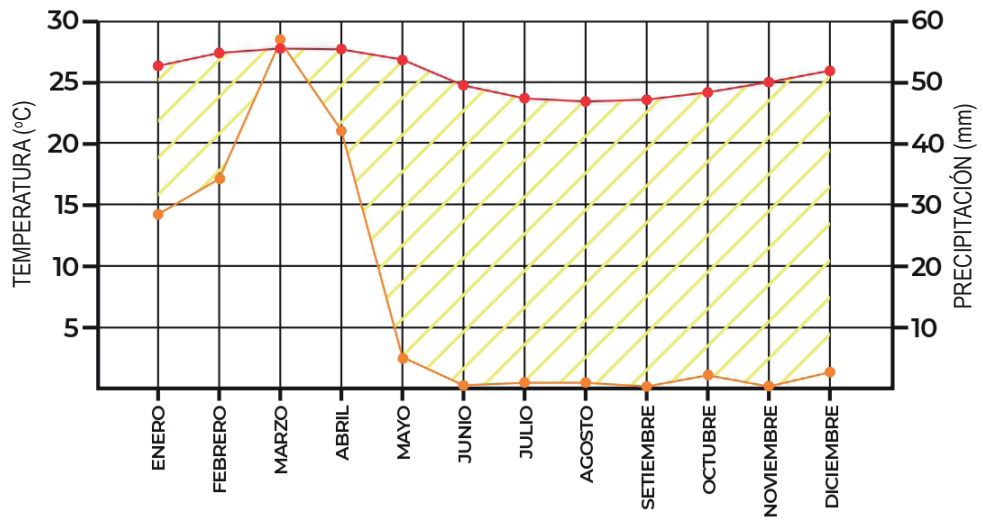

LEYENDA

TEMPERATURA

PRECIPITACIONES

TEMPEPATURA

Cuadro de confort

Zona de confort y estrategias sugeridas

V Zona de confort posible con ventilación ( $\mathrm{v}=2 \mathrm{~m} / \mathrm{s}$

IV Zona de control posible con inercia en verano

IN Zona de control posible con inercia en invierno

ED Zona de control posible con refrigeración evaporativa directa

EI Zona de control posible con refrigeración evaporativa indirecta
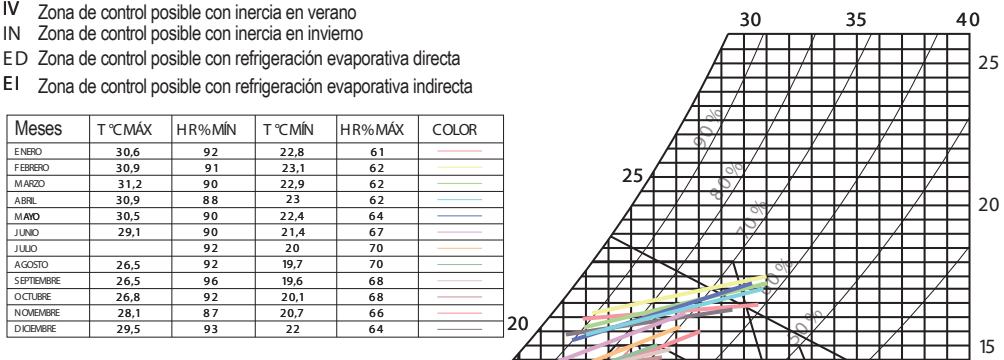

40

25

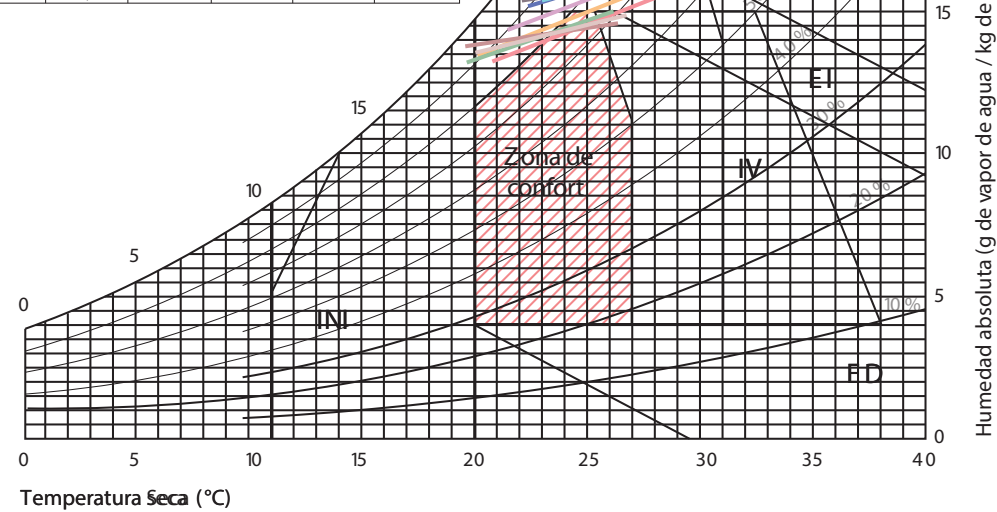



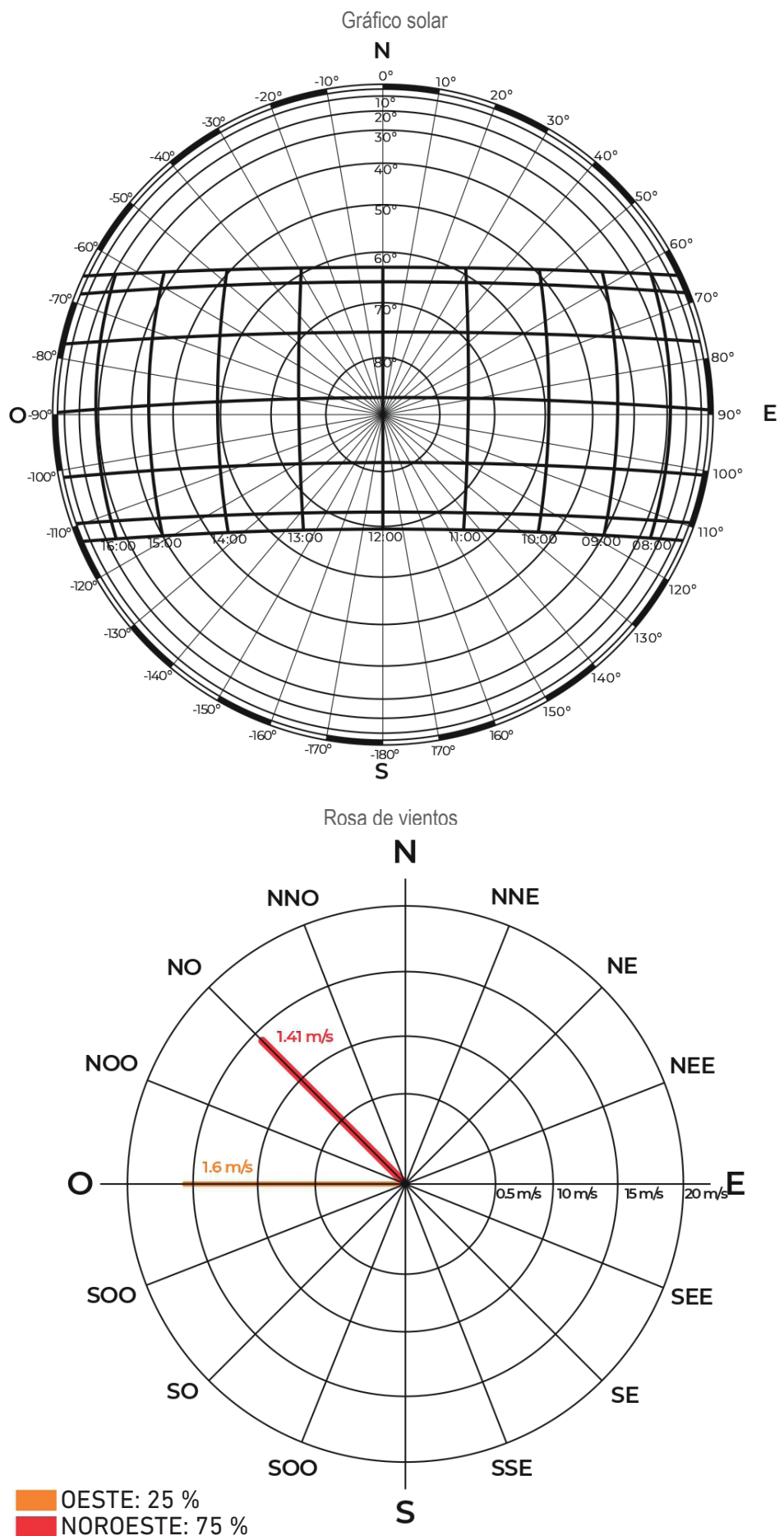

Figura 2

Gráficos

ombrotérmico, de

confort, solar y

rosa de vientos

Elaboración propia 
El gráfico de confort bioclimático de Givoni es quizás el más complejo, pero el que más ayuda para definir las estrategias de diseño para el proyecto. Este gráfico nos permite comparar temperatura seca, humedad absoluta y humedad relativa a lo largo del año para poder caracterizar el clima del lugar y conocer el grado de dificultad para resolver de forma pasiva las necesidades de confort térmico de las personas. El diagrama de Givoni cambia ligeramente según el clima general de la zona: para climas fríos, moderados y cálidos; en nuestro caso, elegimos el diagrama para climas cálidos por las altas temperaturas presentes en Tumbes. La información se coloca tal como detalla Wieser (2011):

Con los datos se terminan construyendo doce líneas, representando cada una de ellas el día típico de cada mes. Cada una de estas líneas está dibujada a partir de los dos momentos del día (puntos extremos que definen la línea) en que se dan las temperaturas máximas y mínimas. Las máximas, alrededor de las 13:00 o 14:00 horas, coinciden con las humedades relativas mínimas, mientras las temperaturas mínimas coinciden con las humedades relativas máximas. (p. 25)

Además de la zona de confort, el diagrama de Givoni señala otras cinco zonas; cada una es el tipo de respuesta pasiva que se puede utilizar para alcanzar el confort térmico. En nuestro caso, vemos que la mayor parte de las líneas se encuentra por encima de la zona de confort, pero muy cerca de esta; el área donde se sitúan nos dice que es posible alcanzar el confort térmico mediante la ventilación del edificio.

El gráfico solar contiene una proyección esférica del recorrido por meses y horas. Nos da un patrón del recorrido solar y nos permite hallar la posición específica del sol en cualquier momento del año. Este gráfico será útil para analizar la iluminación natural dentro de los ambientes del edificio y garantizar la iluminación natural o sombra según se necesite.

En el grafico de la rosa de vientos se coloca la velocidad, orientación y frecuencia del viento en la zona usando el promedio de todo el año. En nuestro caso, el viento llega desde el oeste por tres meses y del noroeste por nueve; la línea más gruesa del noroeste representa este $75 \%$ de frecuencia. Esta información servirá para cuando definamos las estrategias de control pasivo.

Además de los cinco cuadros de información, la ficha bioclimática contiene otras tres partes: conclusiones sobre el clima del lugar, recomendaciones de planteamiento urbano para la zona y recomendaciones de diseño para el edificio (véanse las figuras 3 y 4). Estas conclusiones y recomendaciones nos dan una primera idea general de las estrategias y sistemas de control climático pasivo que deberemos utilizar para alcanzar el confort térmico en esta ubicación. Debido a que los resultados del cuadro de confort de Givoni señalaban que ventilar la vivienda es la mejor estrategia para alcanzar el confort térmico, nuestras recomendaciones de planteamiento urbano y de diseño apuntaban a mejorar la ventilación del edificio. 


\section{CONCLUSIONES}

Durante el mes de MARZO se producen las más altas temperaturas $\left(31,2^{\circ} \mathrm{C}\right)$, el porcentaje de humedad suele ser menor, aumentan las precipitaciones y las horas de sol duranteel día.

Latemperaturamínima $\left(19,7^{\circ} \mathrm{C}\right)$ sedaenel mes deAGOSTO, pueshayunmayorporcentaje de humedadymenos horas de sol duranteel día.

Los vientos constantes se dan por el noroeste con una velocidad promedio de $1,41 \mathrm{~m} / \mathrm{s}$; por lo que se considera la orientación más adecuada para poder ventilar en este tipo de clima. Sin embargo, por el oeste suele haber el promedio de vientos más fuertes, pero con menos frecuencia

El clima es cálido con tendencias a humedades altas; seencuentra mayormentefuera delazona de confort, en lazona de control posible conventilación, por locual se debe aprovechar el lugar generando ventilación natural o mecánica constante por medio del diseño.

Se evidencia que hay variación de humedad y temperatura entre el día y la noche, la cual se demuestra, ya que en la madrugada suele ser mayor el porcentaje de humedad; además, suelehaber poca variación de temperatura entre las estaciones.

\section{PLANTEAMIENTO URBANO}

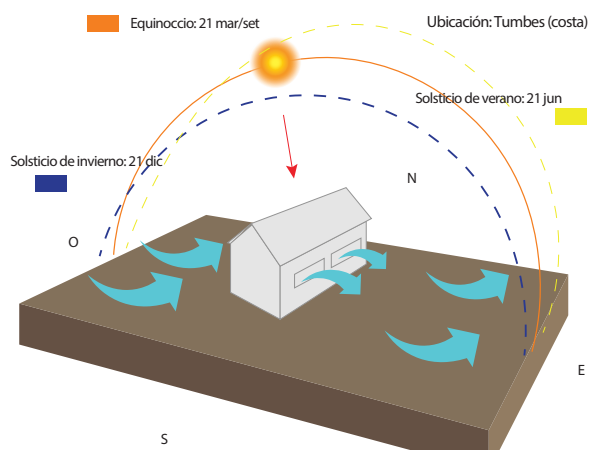

Figura 3.

Conclusiones y

planteamiento urbano

\section{Elaboración propia}

\section{RECOMENDACIONES DE DISEÑO}

Etetechocubrehasta las 16:00 horas.
MARZO, MES MÁS CALUROSO: 14:00 HORAS.
Etetechocubre desdelas 8:00 horas.

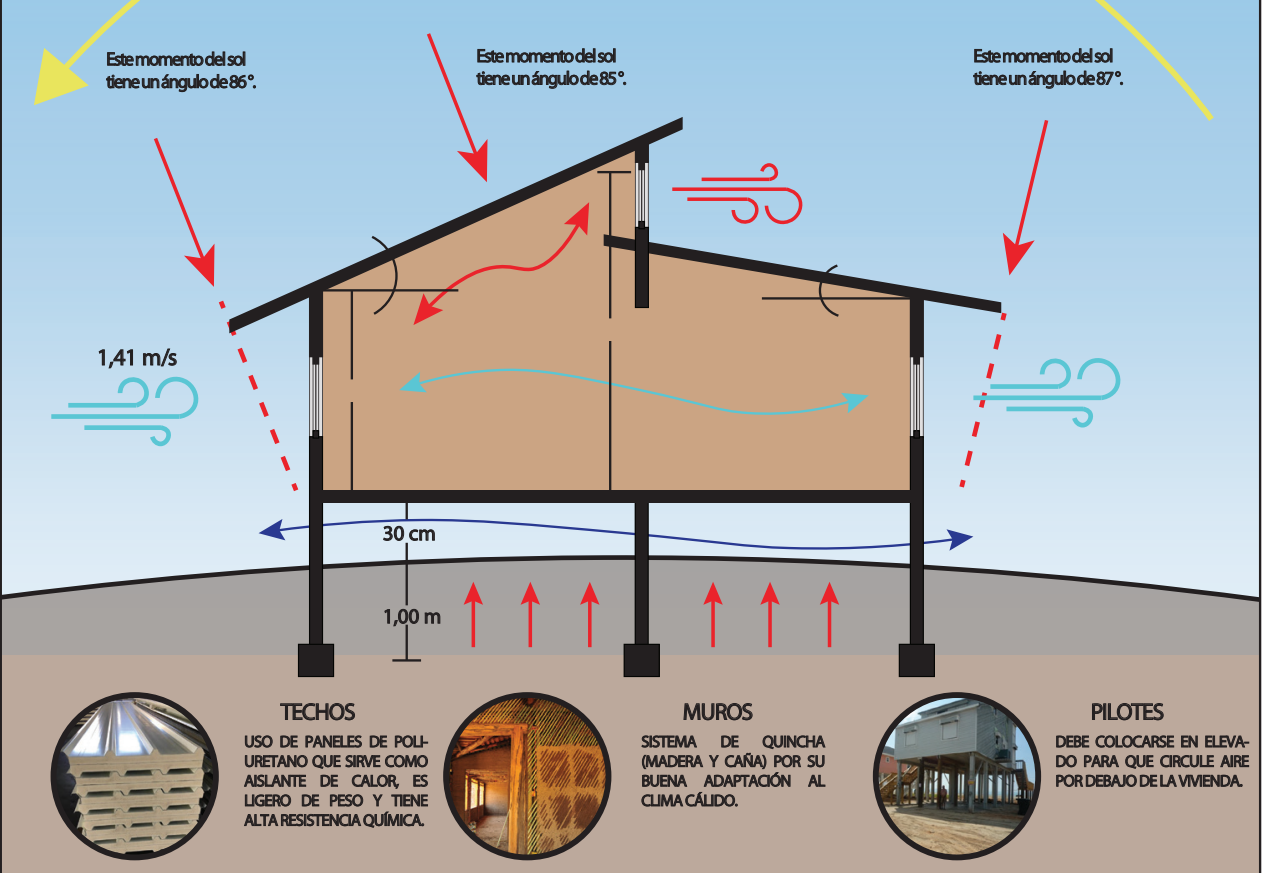

Figura 4.

Recomendaciones de diseño

Elaboración propia 
Respecto al planteamiento urbano, era primordial orientar el edificio hacia el noroeste para aprovechar la mayor frecuencia de vientos desde esa dirección. El asoleamiento se subordina a la búsqueda de la mejor ventilación, ya que, al estar Tumbes ubicado en la latitud $3^{\circ} 34^{\prime} \mathrm{S}$, el punto medio del recorrido solar pasa casi directamente de este a oeste (como se puede ver en el gráfico solar), por lo que la orientación de la vivienda respecto al sol pierde importancia. Como recomendaciones de diseño, inicialmente planteamos cuatro: protección del sol mediante aleros, ventilación cruzada a través del edificio, elevar el edificio del suelo y usar el efecto chimenea para sacar el aire caliente del edificio.

\section{DISEÑO DE LA VIVIENDA BIOCLIMÁTICA}

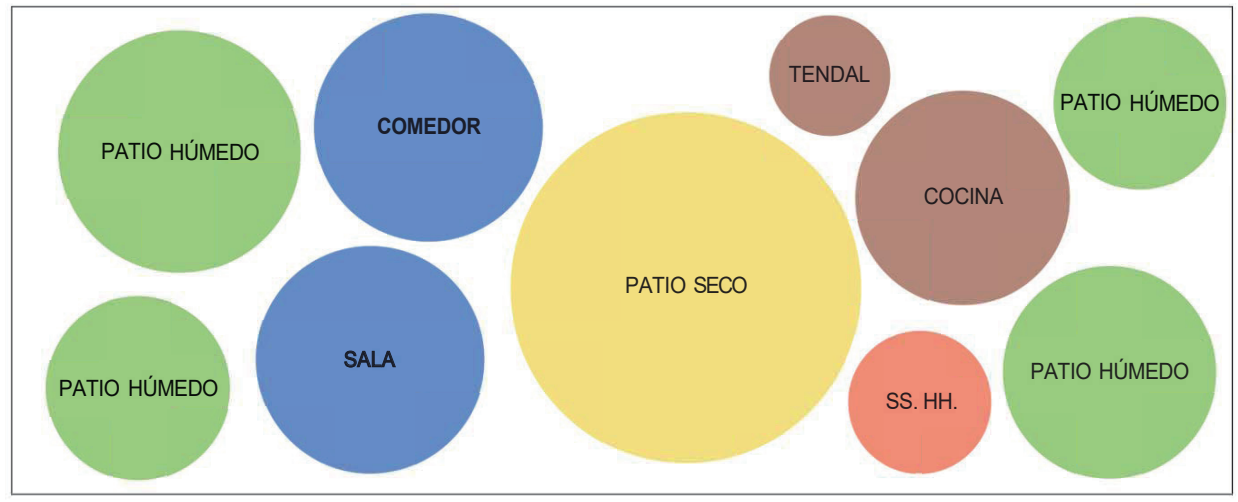

PRIMERA PLANTA

Figura 5 Organigrama de ambientes de la vivienda

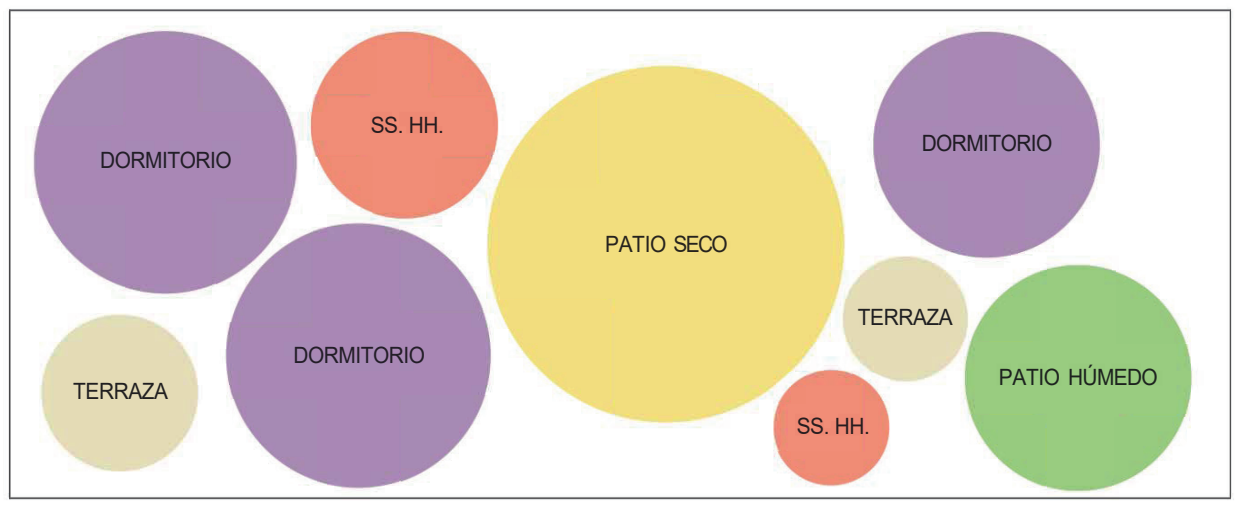

SEGUNDA PLANTA 
Con la información y los criterios obtenidos de la ficha bioclimática pasamos al diseño de la vivienda, teniendo siempre en cuenta las estrategias de control pasivo recomendadas y el buen funcionamiento de la vivienda por igual. Para garantizar el correcto funcionamiento de la vivienda como tal, el primer paso fue establecer un organigrama de los ambientes necesarios y su distribución en planta. En el primer nivel, el acceso es a través del primer patio húmedo hacia la sala, al lado del comedor. El patio seco central separa los ambientes más privados de cocina y lavandería, y el otro patio húmedo. En el segundo nivel, están distribuidos los tres dormitorios con dos baños y dos terrazas. Desde esta primera distribución se puede ver la importancia de las estrategias de control ambiental pasivo en el uso de diferentes tipos de patios. Los patios húmedos laterales enfrían y humedecen el aire que entra a la vivienda hasta llegar al patio seco; en este el aire se calienta y escapa de la vivienda hacia arriba. Esta salida de aire caliente genera un diferencial de presión, lo que mejora la ventilación en el interior del edificio.

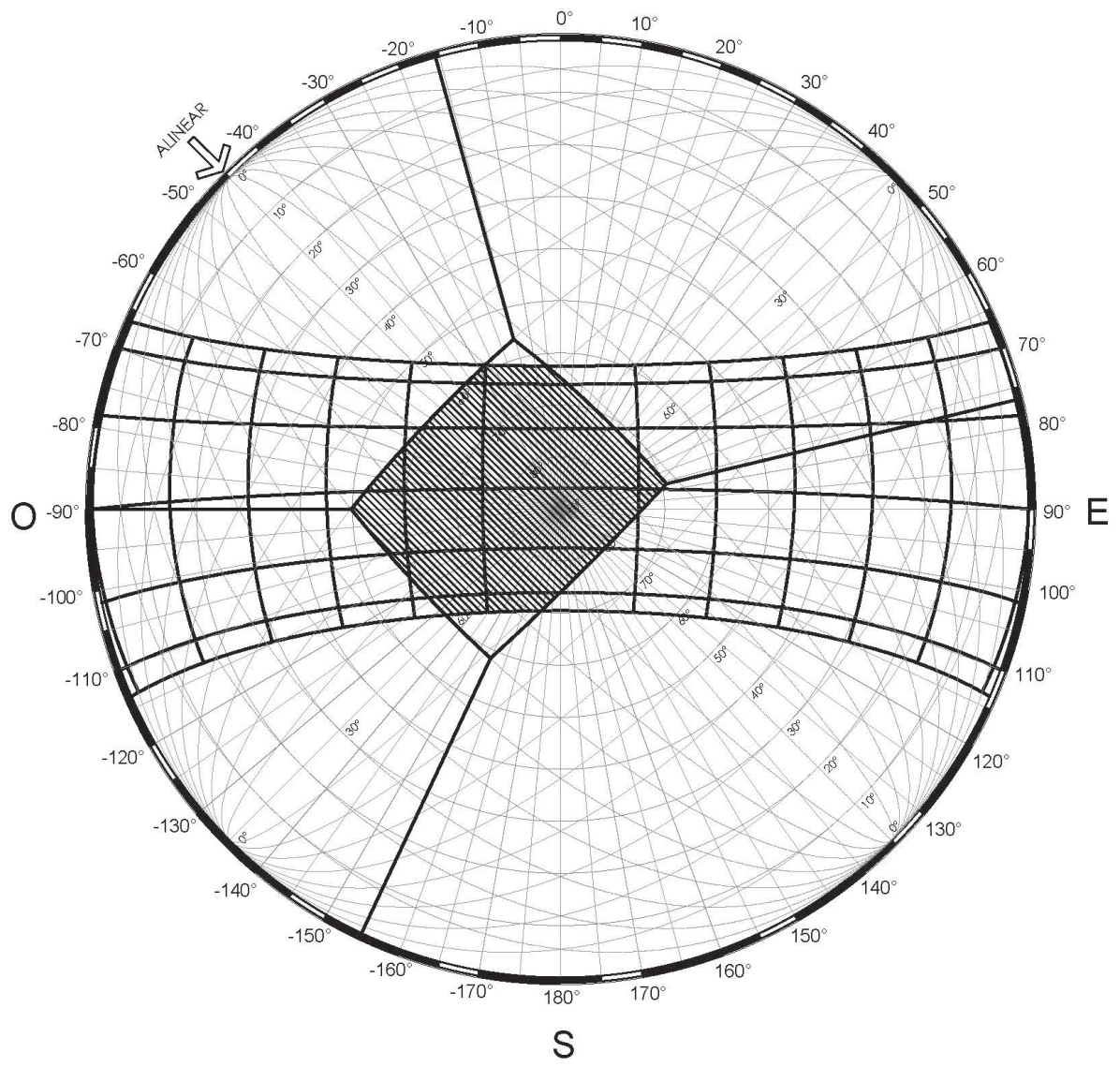

Figura 6. Análisis de incidencia solar en el patio seco 
Siguiendo las recomendaciones de diseño de la ficha bioclimática, en el corte del proyecto se pueden ver los aleros y celosía horizontal de madera en la fachada, que garantizan sombra al patio húmedo, a la sala-comedor y a la terraza de los dormitorios hasta las 3:30 p. m. Esto se logró realizando un análisis de incidencia solar en puntos tanto al interior como al exterior de la vivienda; para este estudio utilizamos el gráfico del recorrido solar colocado en la ficha bioclimática. Por ejemplo, el análisis del punto central del patio seco nos muestra que recibirá sol una hora entre las 12:00 y la 1:00 p. m. durante los solsticios y hasta cuatro horas durante los equinoccios. Con esta información podemos garantizar que el patio cumplirá su función de calentar el aire para que salga de la vivienda. En el corte también se nota la elevación del piso del primer nivel para permitir la circulación de aire bajo este para reducir las temperaturas al interior. En el segundo nivel, los techos altos y en diagonal usan principios del efecto chimenea y de la cámara solar para extraer el aire caliente del ambiente y promover la circulación de aire fresco al interior. Al calentarse la losa de los techos, se genera una zona de aire caliente que escapa rápidamente por las aberturas en la parte más alta del ambiente.

Figura 7 . Diagrama en corte Elaboración propia

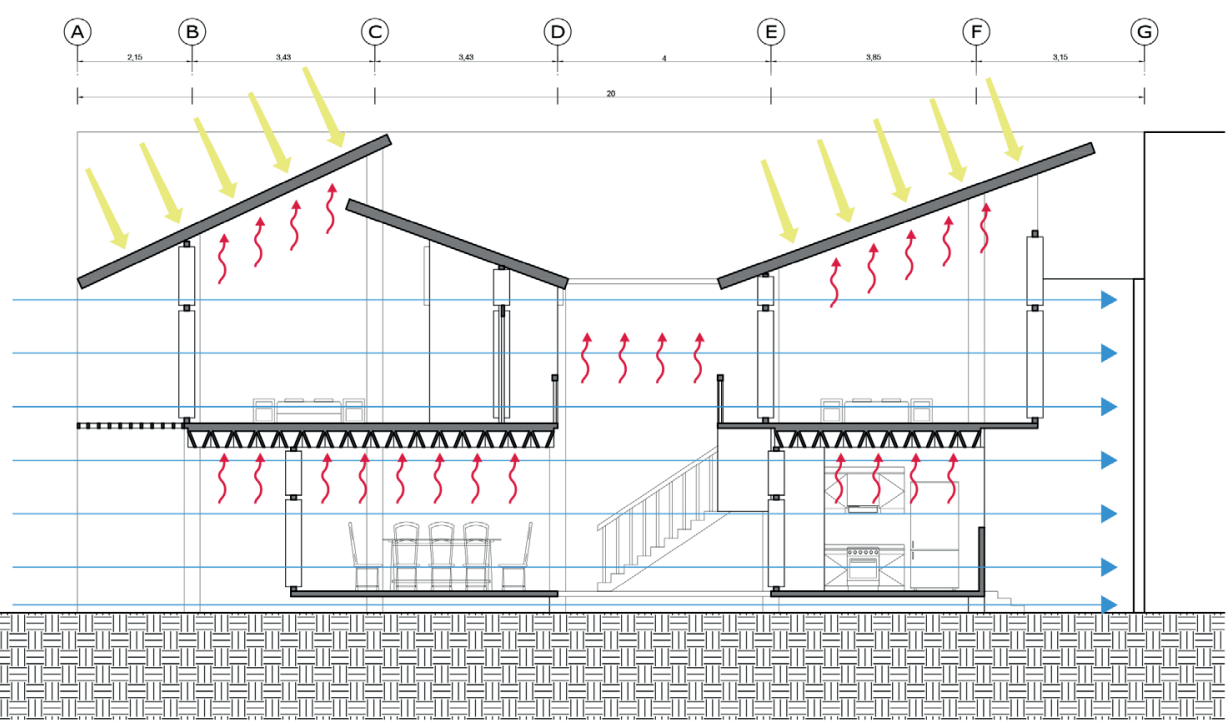

La estrategia utilizada en el nivel superior no es tan fácil de aplicar en el primer nivel, debido a que la losa del segundo nivel debe ser plana; sin embargo, planteamos el uso de una losa prefabricada que facilita la extracción del aire caliente de los ambientes del primer nivel. La losa diseñada por Louis Kahn para la galería de arte de la Universidad de Yale está compuesta por planos inclinados apoyados en tetraedros. Originalmente, el vacío que se crea entre la parte superior de la losa y los tetraedros estaba destinado para las instalaciones eléctricas, sanitarias y ductos de ventilación. En nuestro caso, orientar este vacío hacia el exterior permitiría el ingreso del viento a través de la losa y lo 
aceleraría, por el efecto Venturi, facilitando que empuje hacia los patios el aire caliente que haya subido hacia la losa.
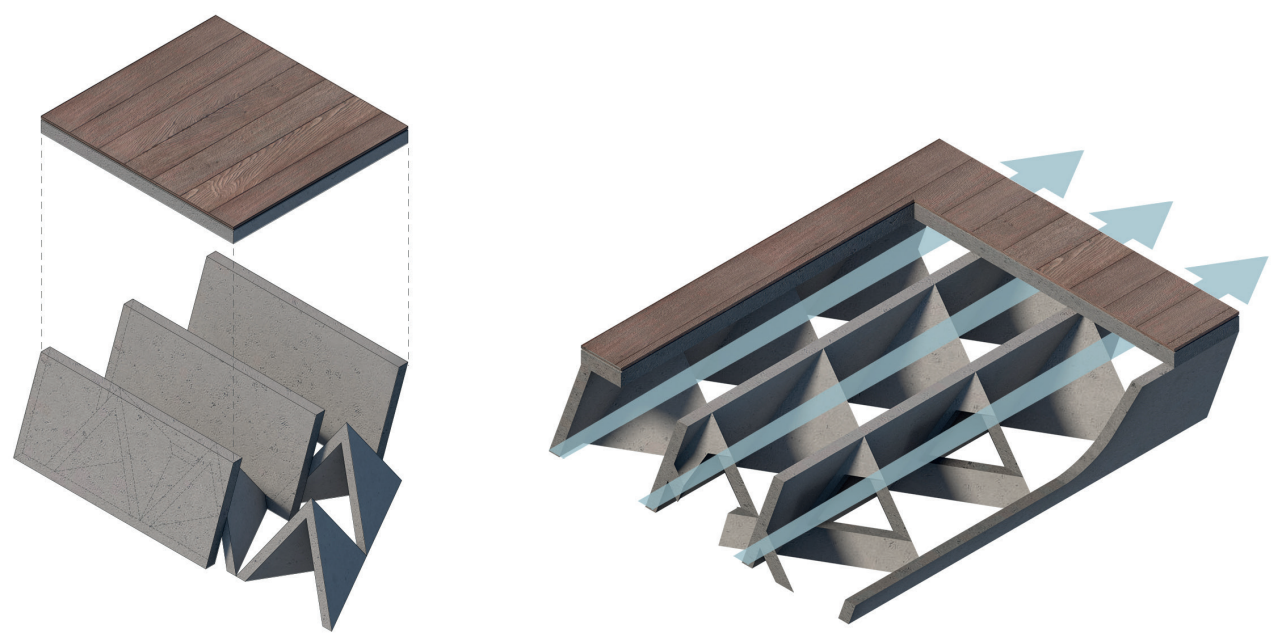

Para los cerramientos planteamos no usar muros, sino celosías móviles de dos cuerpos que podrían configurarse según la necesidad del usuario en determinado momento. Con ambos cuerpos abiertos se permite el ingreso de luz y viento; el cuerpo inferior semicerrado y el superior abierto permite una buena ventilación e iluminación, pero disminuye la incidencia de luz solar directa y aumenta la privacidad; con el cuerpo inferior totalmente cerrado y el superior semicerrado, se permite la ventilación en caso de fuertes lluvias o para mayor privacidad; y ambos cuerpos cerrados, para máxima privacidad y seguridad por las noches o cuando se requiera. Este sistema permite que el viento atraviese la vivienda de lado a lado pasando por las habitaciones sin comprometer totalmente la privacidad de sus usuarios. Por ejemplo, cuando una habitación está vacía, las celosías en su configuración abierta permiten el paso libre del viento; si alguien las usa, cerrar o semicerrar el cuerpo inferior le da privacidad al usuario sin perjudicar la ventilación del resto de ambientes.

$\mathrm{Al}$ aplicarse todas las estrategias de control pasivo previamente mencionadas, ya contamos con un diseño de anteproyecto de esta vivienda bioclimática en la ciudad de Tumbes. Usando diferentes elementos arquitectónicos para garantizar la ventilación de la vivienda, como nos señaló el diagrama de Givoni, garantizamos el confort térmico de sus usuarios. Con un buen diseño eliminamos la necesidad de gastar energía y utilizar sistemas mecánicos, lo que genera un ahorro para los usuarios y disminuye nuestro impacto en el medio ambiente (Garzón, 2007). 
(A)

(B)

(c)

(D)

(E)

(F)
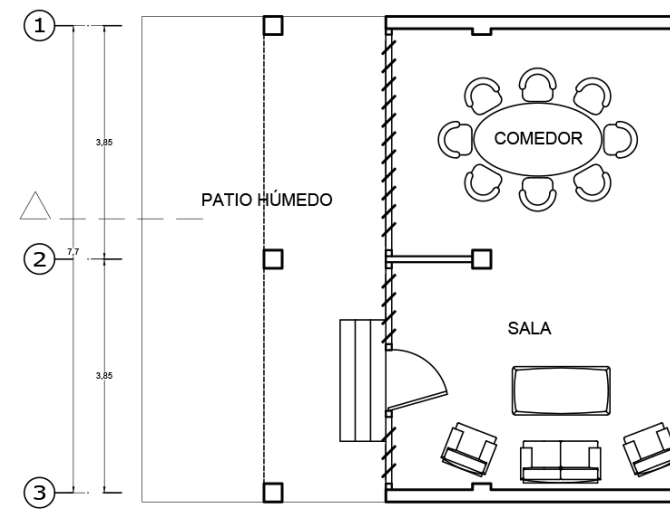

i

17.75

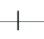

t

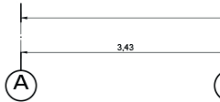

SALA

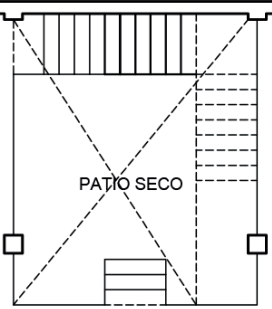

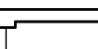
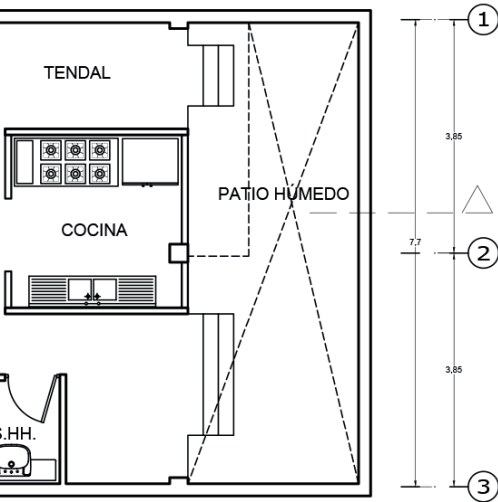

(A)

(B)

(c)

(D)

(E)

(F)

(B)

(C)

(D)

(E)

(F)

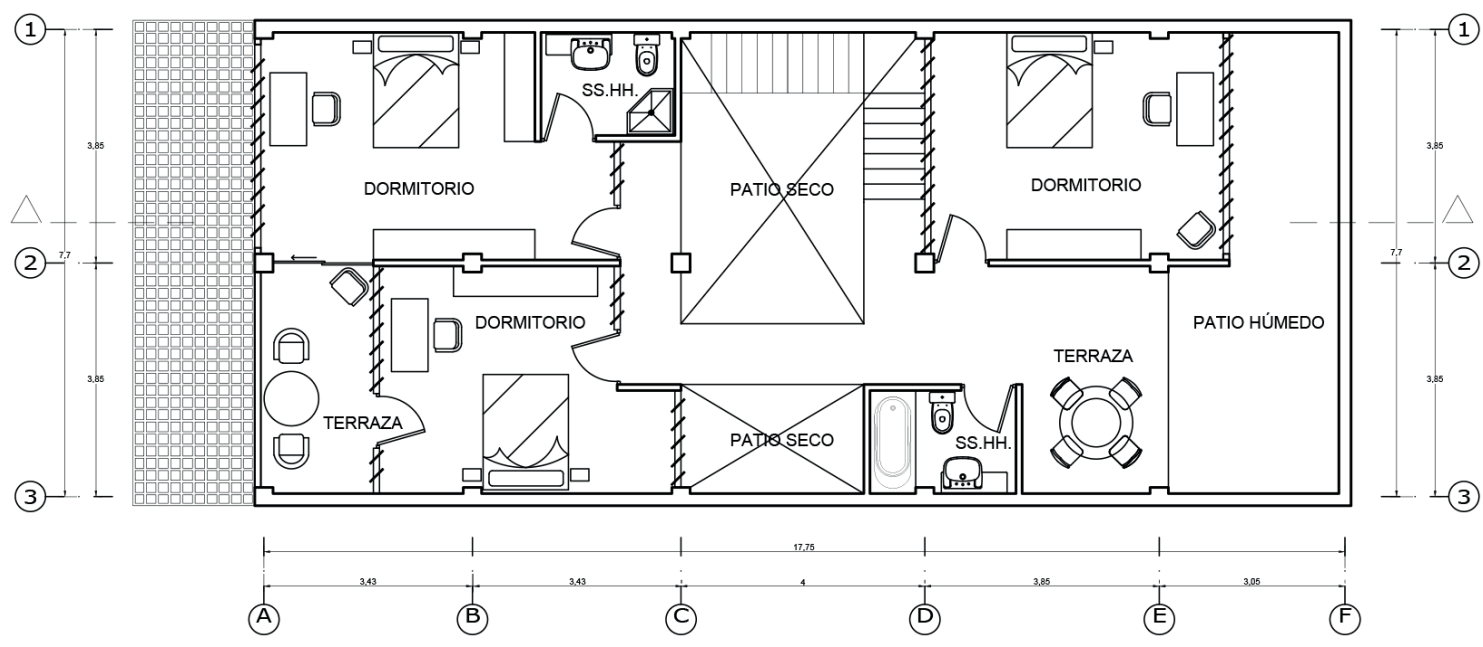

Figura 9.

Plantas de

la vivienda

bioclimática

Elaboración propia 

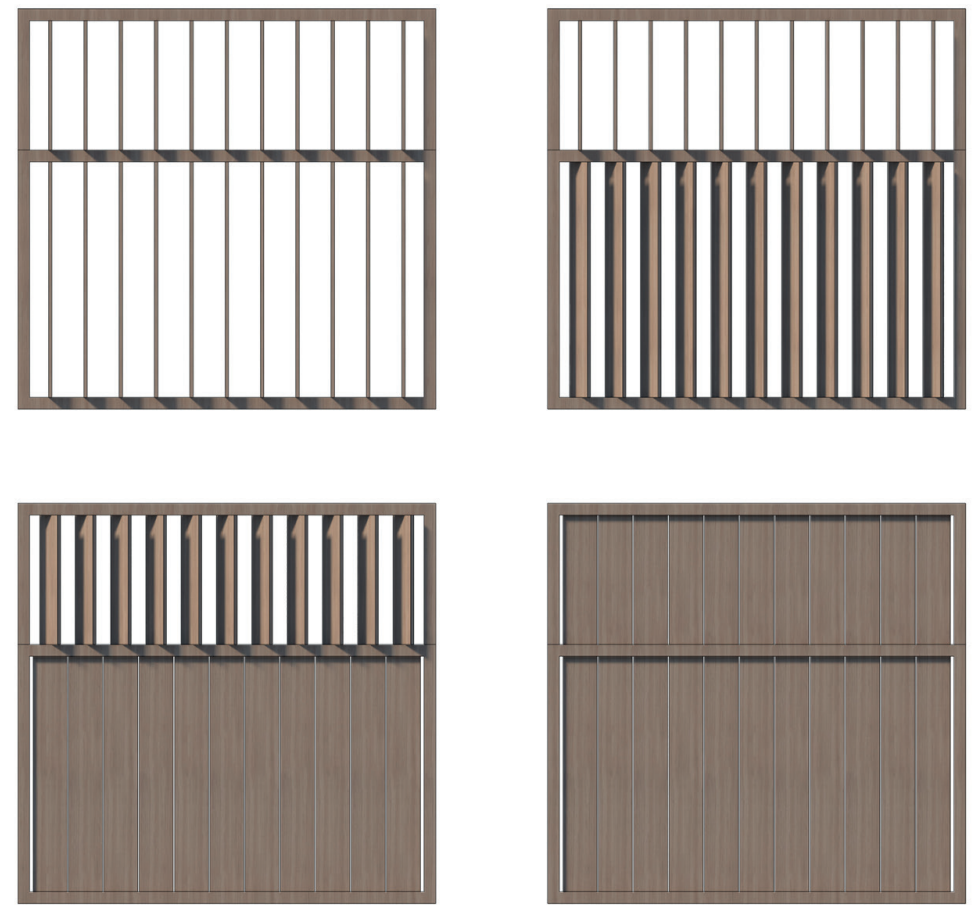

Figura 10.

Configuraciones

de la celosía

Elaboración propia

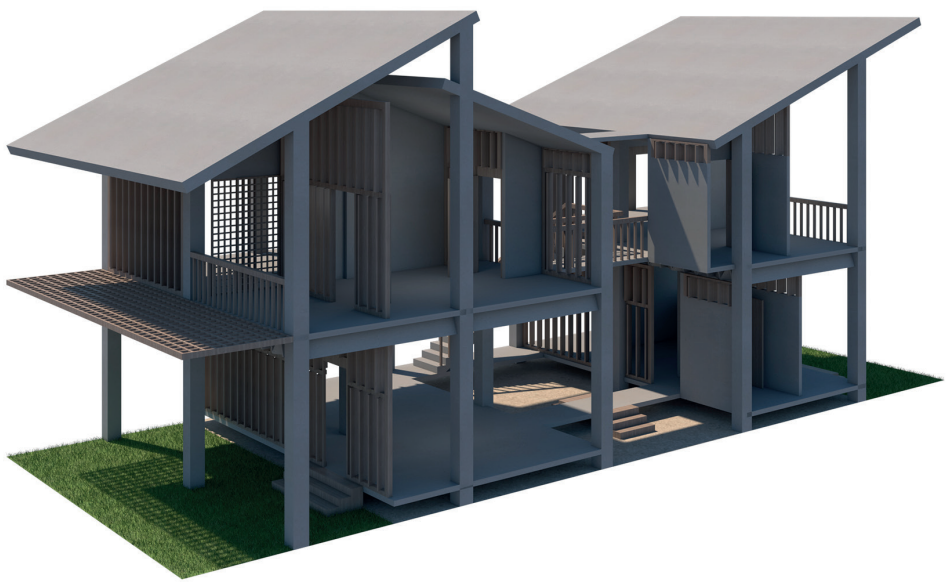

Figura 11 .

Vista 3D de la

vivienda

Elaboración propia 


\section{REFERENCIAS}

Garzón, B. (2007). Arquitectura bioclimática. Nobuko.

Roth, L. M. (1999). Entender la arquitectura: sus elementos, historia y significado. Gustavo Gili.

Wieser, M. (2011). Consideraciones bioclimáticas en el diseño arquitectónico: el caso peruano. Pontificia Universidad Católica del Perú, Departamento de Arquitectura. 